\title{
Predicting the Types of Ion Channel-Targeted Conotoxins Based on AVC-SVM Model
}

\author{
Wang Xianfang, Wang Junmei, Wang Xiaolei, and Zhang Yue \\ School of Computer and Information Engineering, Henan Normal University, Xinxiang 453007, China \\ Correspondence should be addressed to Wang Xianfang; 2wangfang@163.com
}

Received 29 December 2016; Revised 22 February 2017; Accepted 19 March 2017; Published 9 April 2017

Academic Editor: Loris Nanni

Copyright (C) 2017 Wang Xianfang et al. This is an open access article distributed under the Creative Commons Attribution License, which permits unrestricted use, distribution, and reproduction in any medium, provided the original work is properly cited.

\begin{abstract}
The conotoxin proteins are disulfide-rich small peptides. Predicting the types of ion channel-targeted conotoxins has great value in the treatment of chronic diseases, epilepsy, and cardiovascular diseases. To solve the problem of information redundancy existing when using current methods, a new model is presented to predict the types of ion channel-targeted conotoxins based on AVC (Analysis of Variance and Correlation) and SVM (Support Vector Machine). First, the $F$ value is used to measure the significance level of the feature for the result, and the attribute with smaller $F$ value is filtered by rough selection. Secondly, redundancy degree is calculated by Pearson Correlation Coefficient. And the threshold is set to filter attributes with weak independence to get the result of the refinement. Finally, SVM is used to predict the types of ion channel-targeted conotoxins. The experimental results show the proposed AVC-SVM model reaches an overall accuracy of $91.98 \%$, an average accuracy of $92.17 \%$, and the total number of parameters of 68 . The proposed model provides highly useful information for further experimental research. The prediction model will be accessed free of charge at our web server.
\end{abstract}

\section{Introduction}

Conotoxins proteins have many merits, such as low relative molecular mass, stable structure, remarkable activity, high selectivity, and ease of synthesis [1]. Besides, conotoxins have a wide range of applications in the scope of disease treatment, which includes chronic pain, movement disorders, cramps, cancer, and stroke [2]. According to its different targets acting on the organism, the conotoxins can be divided into three categories [3]: (1) acting on voltage-gated ion channels, (2) acting on the ligand-gated ion channel, and (3) acting on other receptors. Further, the voltage-gated ion channels, also known as voltage-sensitive channels, include potassium ion channels, calcium ion channels, and sodium ion channels.

The performance of using different machine learning algorithms in predicting different targets is different. In 2014, neural network and SVM classifier were used to predict lipid binding proteins by Bakhtiarizadeh et al. [4]; the experiments showed that SVM was more successful at discriminating between LBPs and non-LBPs than neural network. In 2016, the potential druggable proteins were predicted through comparing 6 kinds of machine learning algorithms by Jamali et al.; the experiments showed that neural network was the best classifier when predicting potential druggable proteins [5]. In this paper, we will compare the performance of several different machine learning algorithms in the prediction of ion channel types of conotoxin.

There are studies on the prediction of superfamily and family of conotoxins based on protein sequence. In 2006, SVM model was built to predict the superfamily conotoxins based on PseAAC (pseudo amino acid composition) with an overall accuracy of $88.1 \%$ by Mondal et al. [6]. In 2007, an IDQD model was proposed based on dipeptide combinations to predict superfamily and family of conotoxins with accuracy of $87.7 \%$ and $72 \%$, respectively, by Lin and Li [2]. However, there are few researches on the prediction of ion channel types of conotoxins. In 2011, a feature selection approach based ANOVA was used to predict the types of ion channel [7]. In 2013, an RBF model based on the feature selection method of Binomial Distribution was used to predict the ion channels of three types of conotoxins with an overall accuracy of $89.3 \%$ and total of parameters of 70 by Yuan et al. [8]. 
However, these feature extraction methods belong to winding method, which not only depends on the performance of classifier, but also causes time consumption.

In view of the above problems in the prediction of ion channel types of conotoxins, a model named AVC-SVM is proposed based on AVC and SVM in this paper. First, the $F$ value is used to measure the level of significance of all features to the results. Besides, rough selection is carried out to delete the attributes which have less influence on the classification results. Secondly, Pearson Correlation Coefficient $[9,10]$ is introduced to measure the redundancy among the attributes. Then, threshold is set to filter the features whose correlation is too strong. Finally, SVM was used as a classifier to predict the ion channel types of conotoxins. And results of prediction are used to calculate the sensitivity, average precision, and overall accuracy. Results of 5-fold cross-validation show that the AVC-SVM model has better performance when considering accuracy, the total number of features, and running time as a whole.

\section{Preprocessing of Data Sets}

The data sets used in this experiment were derived from Universal Protein Resource (UniProt). In order to obtain a reliable benchmark database, the following steps are performed according to the literature [8]:

(1) Protein sequences must be annotated and evaluated manually.

(2) Protein sequences, which contain ambiguous amino acid residues (such as $\mathrm{X}, \mathrm{B}$, and $\mathrm{Z}$ ), should be excluded.

(3) Amino acid sequences belonging to other protein fragments should be excluded.

(4) Homologous proteins should be excluded.

We used 112 protein sequences as the basic data set which include 24 potassium ion channel-targeted conotoxins, 43 sodium ion channel-targeted conotoxins, and 45 calcium ion channel-targeted conotoxins from [8]. It is necessary to express the protein sequences with the eigenvector of the same number of dimension before predicting [11]. However, the information contained in the eigenvectors tends to be redundant. In the prediction of the ion channel types, the feature selection will directly affect the performance of the classifier [12]. Consequently, it is significant for feature extraction.

\section{Feature Extraction}

The prediction for ion channel types of the conotoxins requires that the protein sequences are represented by the eigenvectors of the same number of dimension. However, there is still redundancy by using general methods of representation of the information. It not only affects the speed of calculation but also affects the results of classification. Therefore, we need to choose the remarkable characteristics of both independence and recognition ability. At present, many feature selection techniques are used to optimize the feature sets, such as ReliefF [13], ReCorre [14], Binomial Distribution [8], and ANOVA [11]. However, few feature selection algorithms have both good prediction accuracy and short running time. In this paper, a novel feature extraction algorithm named AVC is designed to reduce redundancy of attributes and improve the accuracy and speed of prediction.

3.1. Features Representation of Protein Sequences. Both amino acid combinations and dipeptide combinations are often used as parameters for feature selection. The dipeptides combination can not only reflect the information of amino acid residues but also reflect the amino acid sequence number information [7]. Parameters of features by dipeptides combination can reflect the information from protein sequence more comprehensively [2], so we selected dipeptide combinations as parameters to represent features of protein sequences. The total number of dipeptides is 400 ; therefore, there are 400 features. The protein sequence $P$ is defined as follows:

$$
P=\left[a_{1}, a_{2}, \ldots, a_{u}, \ldots, a_{400}\right]
$$

where $a_{u}$ is the frequency of occurrence of the $u$ th dipeptide combination in the protein sequence $P$. The calculation method is shown as follows:

$$
a_{u}=\frac{X_{u}}{\sum_{u} X_{u}} .
$$

In (2), $X_{u}$ is the $u$ th dipeptide in the protein sequence.

Here, we take the protein sequence APELVVTATTTCCGYDPMTICPPCMCTHSCPPKRK as an example; the conversion process is shown in Figure 1.

According to the order of the 20 amino acid residues in the alphabet, we arranged 400 dipeptides. When $u=1$, $a_{1}=f(\mathrm{AA}) \cdot f(\mathrm{AA})$ counts the frequency of occurrence of the dipeptide AA in the protein sequence sample $P$. Similarly, the frequencies of the emergence of 400 dipeptides are obtained from the proteins sequence sample. Finally, the eigenvectors of each protein sequence are decided.

3.2. AVC. The process of the AVC method is described as follows. Firstly, variance-based analysis is used to calculate the ratio $F$ of the variance between groups and variance within the group for each attribute [15]. The size of the $F$ value is used to measure the recognition capability of the attributes [16]. The larger the $F$ value is, the stronger the recognition capability of attribute is [17]. And then the features which have less impact on the results of classification are deleted. Secondly, we introduce Pearson Correlation Coefficient $[9,10]$ to measure the redundancy of attributes. Threshold is set to filter the features whose correlation is too strong. The $F$ value of the $u$ th dipeptide is calculated as follows:

$$
F(u)=\frac{S_{b}^{2}(u)}{S_{w}^{2}(u)},
$$




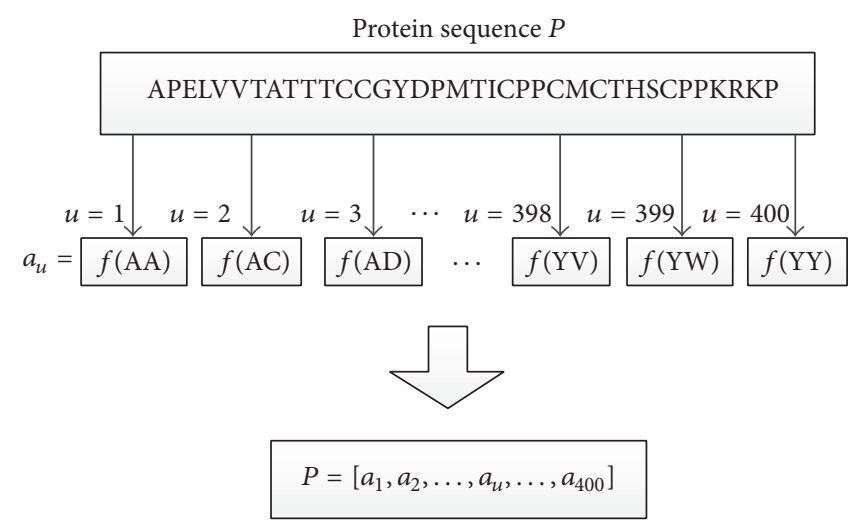

FiguRE 1: Transferring the raw protein sequence to 400 features.

where $S_{b}^{2}(u)$ represents the variance between groups and $S_{w}^{2}(u)$ represents the variance within groups [18]. The calculation methods are shown in (4) and (5), respectively [19]:

$$
\begin{aligned}
S_{b}^{2}(u) & =\frac{S S_{b}(u)}{K-1}, \\
S_{w}^{2}(u) & =\frac{S S_{w}(u)}{N-K},
\end{aligned}
$$

where $K$ is the total of classes and $N$ is the total of samples. Here, the value of $K$ is 3 and the value of $N$ is $112 . S S_{b}(u)$ is the sum of the squares between the groups. And $S_{w}(u)$ is the sum of squares within the groups [20]. The calculation methods are shown in (6) and (7), respectively:

$$
\begin{aligned}
& S S_{b}(u) \\
& \quad=\sum_{i=1}^{K} m_{i}\left(\frac{\sum_{j=1}^{m_{i}} a_{u}(i, j)}{m_{i}}-\frac{\sum_{i=1}^{K} \sum_{j=1}^{m_{i}} a_{u}(i, j)}{\sum_{i=1}^{K} m_{i}}\right)^{2}, \\
& S S_{w}(u)=\sum_{i=1}^{K} \sum_{j=1}^{m_{i}}\left(a_{u}(i, j)-\frac{\sum_{j=1}^{m_{i}} a_{u}(i, j)}{m_{i}}\right)^{2},
\end{aligned}
$$

where $m_{i}$ denotes the total of samples in the $i$ th group (here $m_{1}=24, m_{2}=45$, and $\left.m_{3}=43\right) . a_{u}(i, j)$ represents the frequency of the $u$ th dipeptide of $j$ th samples in the $i$ th group. Take the threshold $f$. If $F(u)<f$, remove $p(u)$ from all samples. Then the rough selection of attributes is completed. The attribute that is not important to the classification result is deleted, and the new feature matrix $P_{x}$ is obtained.

Method of variance-based analysis preserves attributes which have strong recognition ability. However, redundancy may exist in the attributes which have strong recognition ability. It is not conducive to the results of prediction. To solve this problem, Pearson Correlation Coefficient is used to measure correlation between attributes [9]. Its value is between -1 and 1 [10]. We can obtain correlation coefficient between dipeptides. The calculation method is shown as follows:

$$
r_{u v}=\frac{\sum_{i=1}^{N}\left(a_{u}(i)-\overline{a_{u}}\right)\left(a_{v}(i)-\overline{a_{v}}\right)}{(N-1) S_{a_{u}} S_{a_{v}}},
$$

where $a_{u}(i)$ represents occurrence frequency of the $u$ th dipeptide in the $i$ th sample in whole dataset. Similarly, $a_{v}(i)$ represents the frequency of occurrence of the $v$ th dipeptide of the $i$ th sample in whole dataset. $\overline{a_{u}}$ and $\overline{a_{v}}$ are the average of the occurrence frequency of the $u$ th dipeptide and the $v$ th dipeptide in whole dataset, respectively. $S_{a_{u}}$ and $S_{a_{v}}$ are the standard deviation of $a_{u}$ and $a_{v}$, respectively. The calculation method of $S_{a_{u}}$ is shown as follows:

$$
S_{a_{u}}=\sqrt{\frac{\sum_{i=1}^{N}\left(a_{u}(i)-\overline{a_{u}}\right)^{2}}{N-1}} .
$$

The obtained $r_{u v}$ is compared with a preset threshold $r_{0}$. If $r_{u v}>r_{0}$, the correlation between the $v$ th attribute and the $u$ th attribute is larger than the expected value. It means that there is much redundancy between them. And then we compare the $F$ value of the $u$ th with $F$ value of the $v$ th attribute. The attribute whose $F$ value is smaller than another is deleted. We can obtain a collection of attributes which are both strong and independent until all attributes are traversed. A new feature matrix $P_{y}$ is obtained.

\section{Prediction Principle of AVC-SVM}

After feature selection, we need to select an appropriate algorithm to predict the types of ion channels of conotoxins. SVM is a machine learning algorithm based on statistical analysis [21]. It has great advantages in solving nonlinear, small sample and high-dimensional pattern recognition based on the principle of minimizing structural risk [22]. In addition, SVM algorithm also has many applications in bioinformatics $[4,21$, 22]. In this paper, the SVM algorithm was used to predict ion channel types of the conotoxins.

The samples are divided into three categories in this paper. Therefore the method of SVM multiclassification is used to predict the ion channel types of conotoxins. There are many methods of SVM multiclassification such as OVR (oneversus-rest), OVO (one-versus-one), and DAG (Directed Acyclic Graph) [23]. We select OVO method to construct a multiclass classifier to predict the ion channel types of conotoxins. The predictive process using AVC-SVM model is shown in Figure 2. 


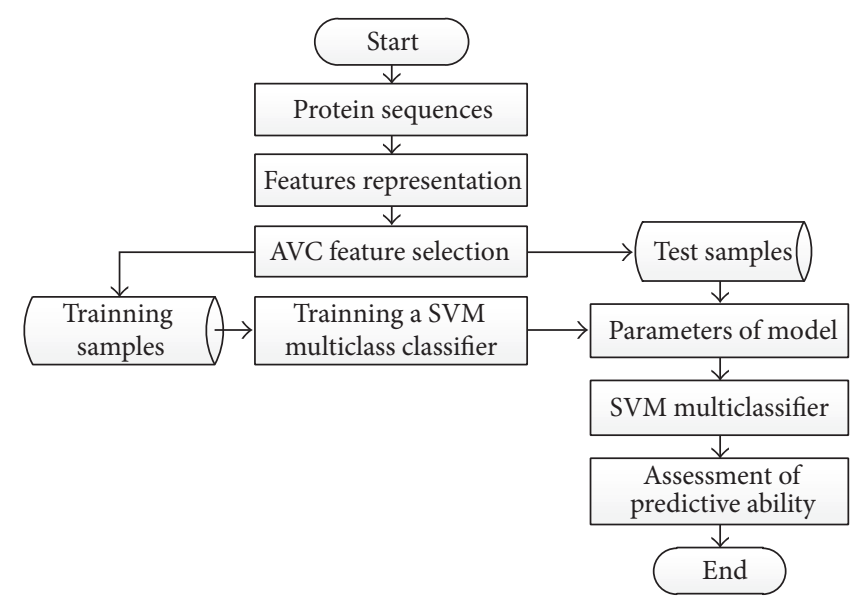

FIGURE 2: The flow chart for prediction of ion channel types of conotoxins by AVC-SVM model.

The principle of method of OVO [24] multiclassification is depicted that there are $k(k-1) / 2$ classifiers for $k$ classes. A classifier is trained for two classes. When classifying an unknown sample, each classifier determines its class and "votes" for the corresponding category. Finally, the category with the largest number of votes is the category of the unknown sample.

4.1. Evaluation Criteria. In the study for the prediction of protein function, the evaluation criteria which are widely used are sensitivity (Sn), overall accuracy (OA), and average accuracy (AA) [25]. They are defined as follows:

$$
\begin{aligned}
\mathrm{Sn}_{i} & =\frac{\mathrm{TP}_{i}}{\left(\mathrm{TP}_{i}+\mathrm{FN}_{i}\right)}, \\
\mathrm{OA} & =\frac{\sum_{i=1}^{n} \mathrm{TP}_{i}}{N}, \\
\mathrm{AA} & =\frac{\sum_{i=1}^{n} \mathrm{Sn}_{i}}{n},
\end{aligned}
$$

where $\mathrm{TP}_{i}$ and $\mathrm{FN}_{i}$ denote true positives and false positives for the $i$ th class, respectively. $N$ and $n$ denote the total of samples and the total of classes, respectively.

4.2. Steps for Prediction. There are five steps to predict the types of ion channels.

Step 1. Formulae (1) and (2) are used to preprocess the date sets and obtain the feature representation of amino acid sequences.

Step 2. The $F$ value calculated by (5) is used to measure the recognition ability of all attributes. Set the threshold $f$. If $F(u)<f$, the $u$ th attribute value $a_{u}$ is deleted from all attributes of samples. And, then, a new vector $P_{x}$ is obtained.

Step 3. Formulae (8) and (9) are used to calculate the correlation coefficient $r_{u v}$ between the $u$ th attribute and the $v$ th attribute in feature matrix $P_{x}$. Set the threshold $r_{0}$; if

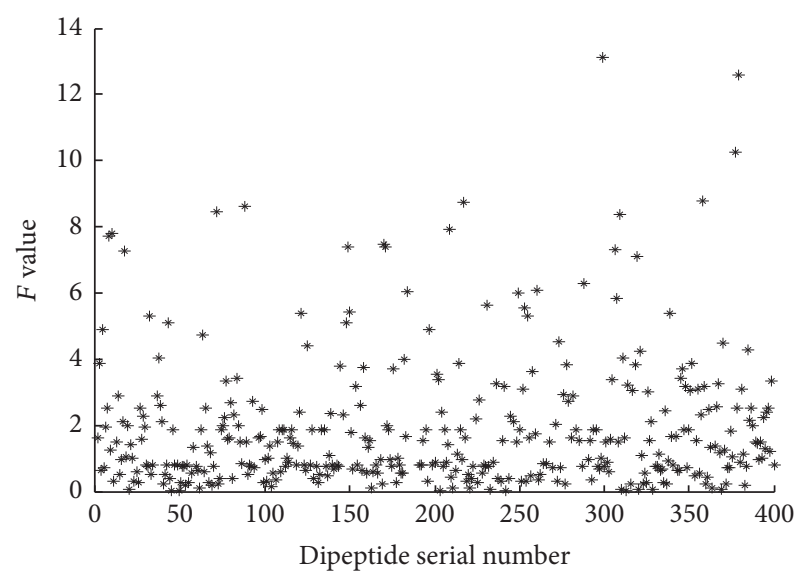

FIGURE 3: Scatter plot of $F$ values for all dipeptides before feature selection.

$r_{u v}>r_{0}, F$ value of the $u$ th attribute is compared with $F$ value of the $v$ th attribute. Then the attribute whose $F$ value is smaller is deleted from the two features.

Step 4. The 112 samples are divided into 5 subsets randomly. One of the five subsets takes turns as test set; the rest are training set. SVM multiclass method was used to train and predict types of ion channel.

Step 5. Formulae (10)-(12) are used to evaluate sensitivity, the overall accuracy, and average accuracy of the model.

\section{Results and Analysis}

5.1. Results of Attributes Reduction Using AVC. The analysis of variance is used to calculate the $F$ values of all the attributes. The distribution of $F$ value of 400 dipeptides is shown in Figure 3. Figures 4 and 5 are the $F$ values of some dipeptides after the rough selection and after the correlation analysis, respectively.

As we can see from Figures 3 and 4, the number of the small $F$ values in Figure 3 is less than that in Figure 4. Because 
TABLE 1: Results of comparison of different feature selection methods.

\begin{tabular}{lccccc}
\hline Methods & $\mathrm{Sn}^{\mathrm{K}}(\%)$ & $\mathrm{Sn}^{\mathrm{Ca}}(\%)$ & $\mathrm{Sn}^{\mathrm{Na}}(\%)$ & $\mathrm{AA}(\%)$ & 92.17 \\
\hline AVC-SVM & 93.14 & 89.21 & 94.17 & 87.79 & 89.87 \\
ANOVA-SVM & 89.28 & 92.54 & 93.3 & 86.8 & 89.25 \\
BiDi-SVM [8] & 83.3 & 83.7 & 76.61 & 83.08 & 87.5 \\
ReliefF-SVM & 87.11 & 85.55 & 82.62 & 78.22 \\
ReCorre-SVM & 78.67 & 73.38 & & 82.25 \\
\hline
\end{tabular}

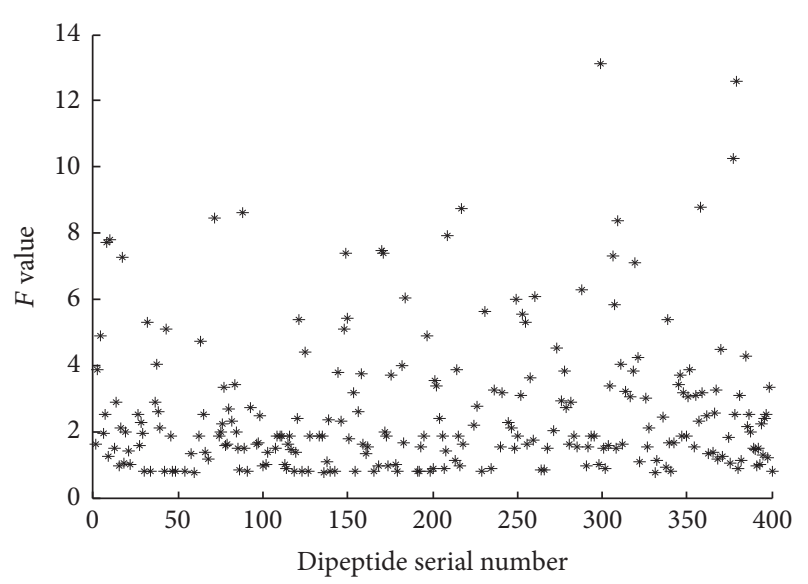

FIGURE 4: Scatter plot of the $F$ value distribution for the portion dipeptides after rough selection.

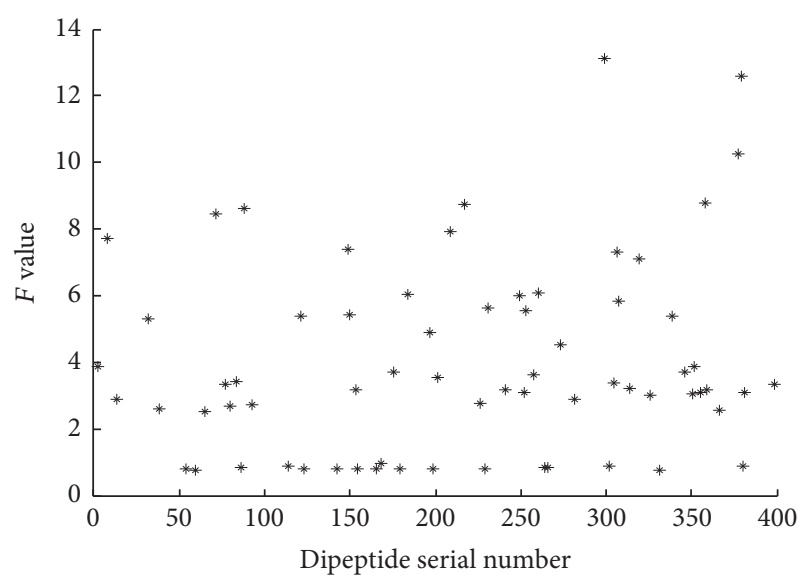

FIGURE 5: Scatter plot of $F$ values for the portion dipeptides after correlation analysis.

the $F$ value measures the ability to identify the attribute, the features which have smaller $F$ value have less effect on the result. Consequently, these attributes are deleted from all features. Figure 5 shows the $F$ value distribution for the portion dipeptides after correlation analysis. The splashes in Figure 5 become few and sparser than the splashes distributed in Figure 4. Figure 5 not only shows the features which have the smaller $F$ value are deleted but also shows that the features having a strong correlation are deleted. It proves that the method of AVC feature selection can reduce the number of dimensions effectively.
TABLE 2: Results of efficiency comparison using different feature selection methods.

\begin{tabular}{lcc}
\hline Methods & Running time $(\mathrm{s})$ & Dimensions \\
\hline AVC-SVM & 0.085 & 68 \\
ANOVA-SVM & 9.350 & 163 \\
BiDi-SVM & 11.939 & 167 \\
ReliefF-SVM & 9.478 & 304 \\
ReCorre-SVM & 7.547 & 99 \\
\hline
\end{tabular}

5.2. Contrastive Results Using Different Methods for Feature Selection. To further illustrate the effectiveness of our method, Table 1 shows the results of comparison of AVC and different feature selection methods. All the classification algorithms in Table 1 use the SVM method and perform 5fold cross-validation.

In Table 1, Sn indicates the sensitivities of three types of ion channels. OA is the overall accuracy. And AA is the average accuracy. The accuracy and sensitivity of the AVC, ANOVA (Analysis of Variance), BiDi (Binomial Distribution) [8], ReliefF [26-28], and ReCorre [14] algorithms are compared when using SVM. The AVC method with an average accuracy of $92.17 \%$ and an overall accuracy of $91.98 \%$ is higher than other methods in Table 1 . In addition, the sensitivities in predicting $\mathrm{K}$ and $\mathrm{Na}$ ion channels using the AVCSVM method are the highest and reach $93.14 \%$ and $94.17 \%$, respectively. The sensitivity using ANOVA method in predicting $\mathrm{Ca}$ ion channel is the best and reaches $92.54 \%$. Comparing the principle of AVC, ANOVA, BiDi, and ReliefF, we can find that only AVC can distinguish the redundant features with strong correlation. Comparing the principle of AVC, ReliefF, and ReCorre, we can find that ReCorre algorithm adds the analysis of relativity analysis based on ReliefF but it does not solve the problem of instability caused by noise and exception points. However, the process of weight calculation based on analysis of variance used in this paper has better robustness. In order to compare the efficiency of feature selection, Table 2 shows running time and the resulting dimensions when using different methods of feature selection. The classification algorithm uses SVM uniformly in Table 2.

The results in Table 2 show the running time of AVCSVM is the shortest and reaches $0.085 \mathrm{~s}$. The running times of ANOVA-SVM, BiDi-SVM, ReliefF-SVM, and ReCorreSVM are $9.350 \mathrm{~s}, 11.939 \mathrm{~s}, 9.478 \mathrm{~s}$, and $7.547 \mathrm{~s}$, respectively. The method with the least dimensions is AVC-SVM with the dimensions of 68 . 
TABLE 3: Results of comparison using different prediction algorithms.

\begin{tabular}{|c|c|c|c|c|c|}
\hline Methods & $\mathrm{Sn}^{\mathrm{K}}(\%)$ & $\mathrm{Sn}^{\mathrm{Ca}}(\%)$ & $\mathrm{Sn}^{\mathrm{Na}}(\%)$ & AA (\%) & OA (\%) \\
\hline AVC-SVM & 93.14 & 89.21 & 94.17 & 92.17 & 91.98 \\
\hline AVC-Bayes & 66.67 & 88.89 & 81.82 & 79.12 & 82.61 \\
\hline AVC-ELM & 59.05 & 79.00 & 90.22 & 76.09 & 78.70 \\
\hline AVC-RF & 75.95 & 79.27 & 79.33 & 78.19 & 76.80 \\
\hline AVC-RBF & 64.67 & 59.91 & 73.59 & 66.05 & 66.09 \\
\hline
\end{tabular}

TABLE 4: Results of comparison using different models.

\begin{tabular}{lccccccc}
\hline Methods & $\mathrm{Sn}^{\mathrm{K}}(\%)$ & $\mathrm{Sn}^{\mathrm{Ca}}(\%)$ & $\mathrm{Sn}^{\mathrm{Na}}(\%)$ & $\mathrm{AA}(\%)$ & $\mathrm{OA}(\%)$ & Dimensions & Running time (s) \\
\hline AVC-SVM & 93.1 & 89.2 & 94.2 & 92.2 & 92.0 & 68 & 0.085 \\
BiDi-RBF [8] & 91.7 & 88.4 & 88.9 & 89.7 & 89.3 & 70 & 11.258 \\
iCTX-Type [30] & 83.3 & 97.8 & 89.8 & 90.3 & 91.1 & 50 & 8.743 \\
F-score-SVM [31] & 91.7 & 95.3 & 95.6 & 94.2 & 94.6 & 180 & 10.594 \\
\hline
\end{tabular}

5.3. Comparison Using Different Multiclassification Algorithms. For the choice of classification algorithm, this paper uses SVM algorithm, which is suitable for the prediction of small sample data [4]. Besides, SVM algorithm does not involve the use of probability measure and law of large numbers, so it is different from the existing statistical methods [29]. In order to prove the superiority of SVM in accuracy and sensitivity, further experiments are needed. When using AVC method to feature selection, the comparisons using different prediction algorithms are shown in Table 3. To make the results more reliable, 5-fold cross-validation was used in all the methods in Table 3.

The results show that AVC-SVM is superior to other methods with the highest average accuracy of $92.17 \%$ and the highest overall accuracy of $91.98 \%$, respectively. The overall accuracies of Bayes [32], ELM (extreme learning machine) [33], RF (Random Forest) [34, 35], and RBF (radial basis function neural network) [36] are $82.61 \%, 78.70 \%, 76.80 \%$, and $66.09 \%$, respectively. Moreover, the sensitivities for the three types of ion channels predicted by SVM are the highest. Comparing SVM with Bayes, ELM, RF, and RBF neural networks, the results show that SVM is the best prediction method when using feature selection of AVC.

5.4. Comparison Using Different Models. In recent years, there are some studies on the prediction of ion channel types of conotoxins. The contrast experiments were shown in Table 4.

It can be seen from Table 4 that AVC-SVM model is better than the BiDi-RBF model and iCTX-Type model in terms of average accuracy, overall accuracy, and time efficiency. When compared with F-score-SVM, the average accuracy and the overall accuracy of the AVC-SVM model are not as high as those in literature [31]. However, the sensitivity of the AVC-SVM model is better than that of the F-score-SVM in predicting $\mathrm{K}$ ion channel. Moreover, the number of features and running time used by the AVC-SVM model is less than the $F$-score-SVM model.

The $F$ value used in our method and $F$-score proposed by the literature [30] are different. The $F$-score in the literature
[30] is the ratio of the variance between groups and the variance within groups. The variance between groups in the literature [30] is calculated using sum of squares of deviations. The $F$ value in our paper is the ratio of the mean square deviation between groups and the mean square deviation within groups. In this paper, the mean square deviation is the sum of squares of deviations divided by degree of freedom. It can eliminate the impact caused by imbalance of number of samples between groups.

\section{Conclusions}

In this paper, the proposed model based on feature selection of AVC and prediction method of SVM is used to predict the type of ion channels. The results of 5-fold cross-validation show that our model reaches high predicted accuracies and the feature selection method in this paper has two advantages over other feature selection methods: first, the analysis of correlation for features is used to further reduce the existing information redundancy between the strong correlating features. Second, the calculated process for weights of the attributes is robust. However, it is necessary to declare the data set which is mined for analysis. We will further expand the data set in the follow-up work for in-depth analysis.

\section{Conflicts of Interest}

The authors declare that they have no conflicts of interest.

\section{Acknowledgments}

This work was supported by the National Natural Science Foundation of China (no. 61173071), the Science and Technology Research Project of Henan Province (no. 122102210079), the 2013 Program of China Scholarship Council Countries about Senior Research Scholar and Visiting Scholar (no. 201308410018), the Innovation Talent Support Program of Henan Province Universities (no. 2012HASTIT011), the Doctoral Started Project of Henan Normal University (no. 1039), and the International Training Project of High-Level Talents 
(no. 17) of Henan Administration of Foreign Experts Affairs in 2016. Therefore, it is necessary for the stability conditions to be investigated in the multiregions.

\section{References}

[1] S. D. Robinson and R. S. Norton, "Conotoxin gene superfamilies," Marine Drugs, vol. 12, no. 12, pp. 6058-6101, 2014.

[2] H. Lin and Q.-Z. Li, "Predicting conotoxin superfamily and family by using pseudo amino acid composition and modified Mahalanobis discriminant," Biochemical and Biophysical Research Communications, vol. 354, no. 2, pp. 548-551, 2007.

[3] M. E. Williams, P. F. Brust, D. H. Feldman et al., "Structure and functional expression of an $\omega$-conotoxin-sensitive human $\mathrm{N}$ type calcium channel," Science, vol. 257, no. 5068, pp. 389-395, 1992.

[4] M. R. Bakhtiarizadeh, M. Moradi-Shahrbabak, M. Ebrahimi, and E. Ebrahimie, "Neural network and SVM classifiers accurately predict lipid binding proteins, irrespective of sequence homology," Journal of Theoretical Biology, vol. 356, pp. 213-222, 2014.

[5] A. A. Jamali, R. Ferdousi, S. Razzaghi, J. Li, R. Safdari, and E. Ebrahimie, "DrugMiner: comparative analysis of machine learning algorithms for prediction of potential druggable proteins," Drug Discovery Today, vol. 21, no. 5, pp. 718-724, 2016.

[6] S. Mondal, R. Bhavna, R. Babu, and S. Ramakumar, "Pseudo amino acid composition and multi-class support vector machines approach for conotoxin superfamily classification," Journal of Theoretical Biology, vol. 243, no. 2, pp. 252-260, 2006.

[7] H. Lin and H. Ding, "Predicting ion channels and their types by the dipeptide mode of pseudo amino acid composition," Journal of Theoretical Biology, vol. 269, pp. 64-69, 2011.

[8] L.-F. Yuan, C. Ding, S.-H. Guo, H. Ding, W. Chen, and H. Lin, "Prediction of the types of ion channel-targeted conotoxins based on radial basis function network," Toxicology in Vitro, vol. 27, no. 2, pp. 852-856, 2013.

[9] A. de Miranda Neto, "Pearson's correlation coefficient: a more realistic threshold for applications on autonomous robotics," Computer Technology and Application, vol. 5, no. 2, pp. 69-72, 2014.

[10] V. J. DeGhett, "Effective use of Pearson's product-moment correlation coefficient: an additional point," Animal Behaviour, vol. 98, pp. el-e2, 2014.

[11] H. Lin, W.-X. Liu, J. He, X.-H. Liu, H. Ding, and W. Chen, "Predicting cancerlectins by the optimal g-gap dipeptides," Scientific Reports, vol. 5, Article ID 16964, 2015.

[12] H. Lin and W. Chen, "Prediction of thermophilic proteins using feature selection technique," Journal of Microbiological Methods, vol. 84, no. 1, pp. 67-70, 2011.

[13] Y. Zhang, C. Ding, and T. Li, "Gene selection algorithm by combining reliefF and mRMR," BMC Genomics, vol. 9, no. 2, article S27, 2008.

[14] L. Zhang, J. Wang, Y. Zhao et al., "Combination feature selection based on relief," Journal of Fudan University (Natural Science Edition), vol. 43, no. 5, pp. 893-898, 2004.

[15] H. Ding and D. Li, "Identification of mitochondrial proteins of malaria parasite using analysis of variance," Amino Acids, vol. 47, no. 2, pp. 329-333, 2015.

[16] H. Ding, S.-H. Guo, E.-Z. Deng et al., "Prediction of Golgiresident protein types by using feature selection technique,"
Chemometrics and Intelligent Laboratory Systems, vol. 124, pp. 9-13, 2013.

[17] H. Ding, P.-M. Feng, W. Chen, and H. Lin, "Identification of bacteriophage virion proteins by the ANOVA feature selection and analysis," Molecular BioSystems, vol. 10, no. 8, pp. 22292235, 2014.

[18] M. J. Anderson and C. J. ter Braak, "Permutation tests for multifactorial analysis of variance," Journal of Statistical Computation and Simulation, vol. 73, no. 2, pp. 85-113, 2003.

[19] E. A. Rady, N. M. Kilany, and S. A. Eliwa, "Estimation in mixedeffects functional ANOVA models," Journal of Multivariate Analysis, vol. 133, pp. 346-355, 2015.

[20] F. Chen, Z. Li, L. Shi, and L. Zhu, "Inference for mixed models of ANOVA type with high-dimensional data," Journal of Multivariate Analysis, vol. 133, pp. 382-401, 2015.

[21] B.-C. Kuo, H.-H. Ho, C.-H. Li, C.-C. Hung, and J.-S. Taur, "A kernel-based feature selection method for SVM with RBF kernel for hyperspectral image classification," IEEE Journal of Selected Topics in Applied Earth Observations and Remote Sensing, vol. 7, no. 1, pp. 317-326, 2014.

[22] C. Hou, F. Nie, C. Zhang, D. Yi, and Y. Wu, "Multiple rank multilinear SVM for matrix data classification," Pattern Recognition, vol. 47, no. 1, pp. 454-469, 2014.

[23] G. Madzarov, D. Gjorgjevikj, and I. Chorbev, "A multi-class SVM classifier utilizing binary decision tree," Informatica, vol. 33, no. 2, pp. 225-233, 2009.

[24] M. Galar, A. Fernández, E. Barrenechea, and F. Herrera, "DRCW-OVO: distance-based relative competence weighting combination for One-vs-One strategy in multi-class problems," Pattern Recognition, vol. 48, no. 1, pp. 28-42, 2015.

[25] H. Lin, H. Ding, F.-B. Guo, and J. Huang, "Prediction of subcellular location of mycobacterial protein using feature selection techniques," Molecular Diversity, vol. 14, no. 4, pp. 667671,2010

[26] Y. Huang, P. J. McCullagh, and N. D. Black, "An optimization of ReliefF for classification in large datasets," Data and Knowledge Engineering, vol. 68, no. 11, pp. 1348-1356, 2009.

[27] J. Ghasemian, M. Moallem, Y. Alipour et al., "Predicting students' grades using fuzzy non-parametric regression method and ReliefF-based algorithm," Advances in Computer Science, vol. 3, no. 2, pp. 43-51, 2014.

[28] A. Zafra, M. Pechenizkiy, and S. Ventura, "ReliefF-MI: an extension of ReliefF to multiple instance learning," Neurocomputing, vol. 75, pp. 210-218, 2012.

[29] M. Lapin, M. Hein, and B. Schiele, "Learning using privileged information: SVM+ and weighted SVM," Neural Networks, vol. 53, pp. 95-108, 2014.

[30] H. Ding, E.-Z. Deng, L.-F. Yuan et al., "ICTX-type: a sequencebased predictor for identifying the types of conotoxins in targeting ion channels," BioMed Research International, vol. 2014, Article ID 286419, 2014.

[31] Y. Wu, Y. Zheng, and H. Tang, "Identifying the types of ion channel-targeted conotoxins by incorporating new properties of residues into pseudo amino acid composition," BioMed Research International, vol. 2016, Article ID 3981478, 5 pages, 2016.

[32] Z.-L. Xiang, X.-R. Yu, and D.-K. Kang, "Experimental analysis of naïve Bayes classifier based on an attribute weighting framework with smooth kernel density estimations," Applied Intelligence, vol. 44, no. 3, pp. 611-620, 2016. 
[33] Z. Bai, G.-B. Huang, D. Wang, H. Wang, and M. B. Westover, "Sparse extreme learning machine for classification," IEEE Transactions on Cybernetics, vol. 44, no. 10, pp. 1858-1870, 2014.

[34] Q. Wu, Y. Ye, H. Zhang, M. K. Ng, and S.-S. Ho, "ForesTexter: an efficient random forest algorithm for imbalanced text categorization," Knowledge-Based Systems, vol. 67, pp. 105-116, 2014.

[35] M. Saraswat and K. V. Arya, "Feature selection and classification of leukocytes using random forest," Medical and Biological Engineering and Computing, vol. 52, no. 12, pp. 1041-1052, 2014.

[36] T. Xiong, Y. Bao, Z. Hu, and R. Chiong, "Forecasting interval time series using a fully complex-valued RBF neural network with DPSO and PSO algorithms," Information Sciences, vol. 305, pp. 77-92, 2015. 

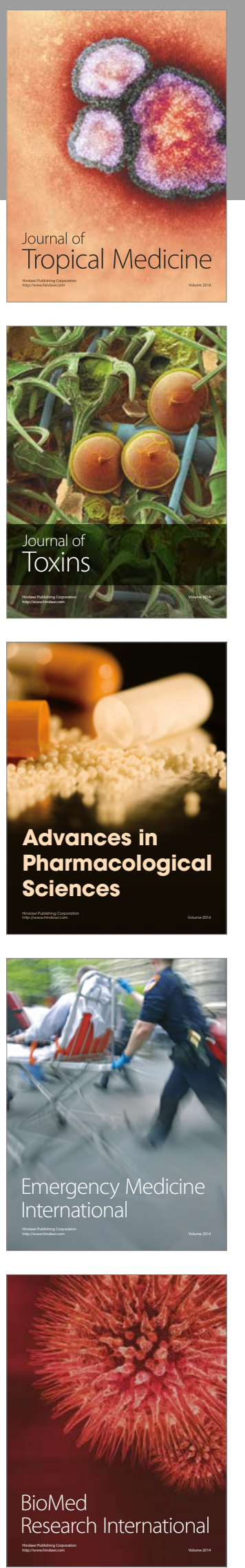
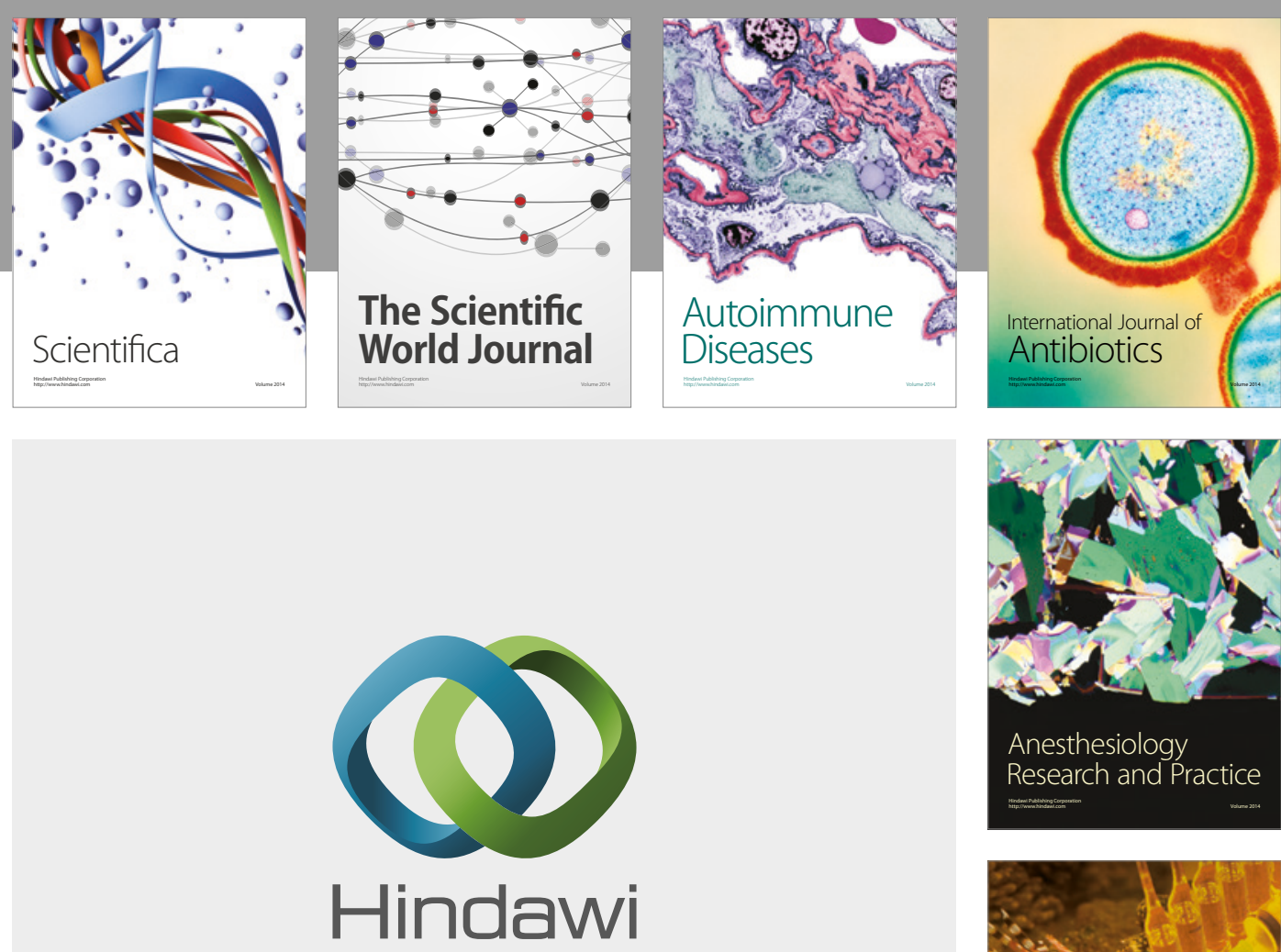

Submit your manuscripts at

https://www.hindawi.com
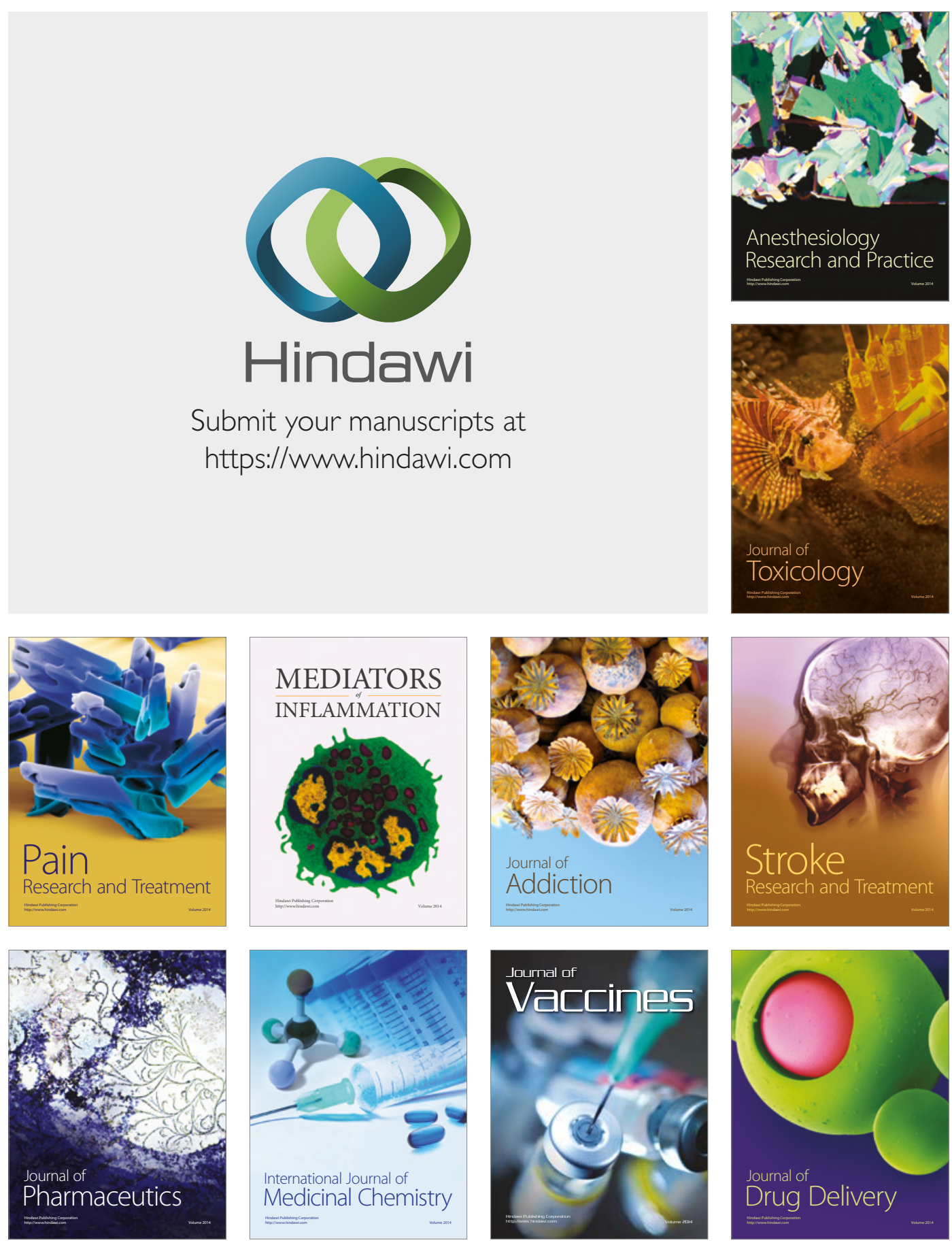